\title{
Mini-implants, mega solutions: a review
}

\author{
Alaveni Manga Narsingoju 1,*, C. Ravi Kumar², Harilal G33, Pavani Lukka4 \\ ${ }^{1}$ Postgraduate Student, ${ }^{2}$ Professor, ${ }^{3}$ Reader, ${ }^{4}$ Senior Lecturer, Department of Prosthodontics, Mamata \\ Dental College, Giri Prasad Nagar, Khammam, Telangana, India -507002.
}

\section{N F O R M A T I O N A B S T R A C T}

\section{Article History}

Received 10th April 2021

Received revised

$5^{\text {th }}$ July 2021

Accepted $13^{\text {th }}$ July 2021

Available online

$1^{\text {st }}$ August 2021

\section{K E Y W O R D S}

Mini-implants

Overdenture

Splinting

Osteotomy
Dental implants have evolved as a standard treatment option for the replacement of missing teeth. Though this treatment modality provides a high level of patient satisfaction and success, it cannot be performed in all cases. Implant use is also restricted when the quality and quantity of bone at the edentulous site is limited, in addition to medically impaired patients. Among the conditions are remaining ridges with reduced interdental spacing, atrophic edentulous maxillary and mandibular ridges, and narrow ridges such as the mandibular incisor and maxillary lateral incisor area. A proper augmentation method for the placement of a regular diameter implant (3.75 to $7 \mathrm{~mm}$ ) can improve the height and width of bone at such sites. However, bone augmentation and bone grafting procedures are rarely undertaken due to financial constraints, the risk of subjecting the patient to additional surgical procedures, the added time factor, or the guarded prognosis of the grafted site. In such cases, mini-implants are the choice of treatment. Mini-implants have the potential to be a viable alternative to standarddiameter implants in some circumstances. Benefits of mini-implants can be gained by replacing a single missing tooth, or preferably they must be used in multiples to retain fixed dental prostheses and might serve as an inexpensive, and efficient solution for retaining overdentures in selected cases.

\section{Introduction}

A prosthodontist's primary goal is to restore function and esthetics by replacing lost teeth. The high success rate of dental implant therapy has been a major factor in restoring function and esthetics effectively. In distal extension, the placement of long-span bridges and implants are standard treatment modalities in fixed prosthodontics. However, conventional implants are not recommended for patients having ridges with less buccolingual [1], and mesiodistal width [2]. Further, interproximal bone loss may be observed when the distance between the implant and the adjacent tooth is less than $1.5 \mathrm{~mm} \mathrm{[3].}$

The standard diameter of endosseous implants ranges between 3.75 to $7 \mathrm{~mm}$ [4] If they ought to be used in narrow ridges, bone grafting, ridge augmentation, and ridge expansion procedures are mandatory. Bone grafting, ridge augmentation, ridge expansion procedures are complex procedures, and the prognosis of bone grafting procedures is questionable [4]. The stabilization of implants mainly depends on the cortical bone. However, studies have shown the formation of trabecular bone in the grafted area, and there is no evidence of the maturation of grafted bone to cortical bone [4]. Therefore, in narrow ridges, mini-implants have become an alternative to conventional methods include bone grafting, ridge augmentation, and ridge expansion procedures [4]. Small diameter implants are 
available in a range from 3.0 to $3.3 \mathrm{~mm}$ and very small or "mini" 1.8 to $3 \mathrm{~mm}$ diameter implants [5]. Miniimplants are indicated in patients with reduced interradicular bone, narrow ridges, or reduced mesiodistal prosthetic space [6]. Multiple mini-implants can be used for removable full or partial denture stabilization [5] and are offered at a lower cost. Mini-implants are indicated in the narrow ridges of edentulous or partially edentulous arches. Especially in the anterior maxilla with a decreased palato-labial bone width and/or insufficient interdental space are present [7]. In the atrophic posterior mandible, inadequate buccolingual bone width is the common problem for implant placement. In these cases, mini-implants are the treatment of choice [7].

\section{Mini-implant design}

Standard implants with a diameter of $3.5 \mathrm{~mm}$ to $7 \mathrm{~mm}$ are available in a one-piece design with a fused abutment or in a two-piece design where the abutment is attached later [7]. Mini-implants manufactured with a diameter of $1.8 \mathrm{~mm}$ to $3 \mathrm{~mm}$ are available in a one-piece design with a ball-shaped head (Figure 1) used for stabilization of removable prosthesis and with a square head for fixed prosthesis. Abutments and screws are not used in mini-implants like they are in conventional implants. In case of mini-implants, in the mini-implants, the prosthetic teeth are held in place by elastic 0-rings (Figure2) on a ball at the top of the implant. In orthodontics, mini-implants are used for indirect anchorage, these implants contain racket like head design. Abutment protrudes over the gingiva when an implant is placed in the bone. The transmucosal component of the neck of a mini-implant's must be smooth and vary in length based on the implant site's mucosal thickness [7-10].

\section{Indications}

Mini dental implants (MDIs) may be a viable treatment option in places with insufficient ridge width and/or interdental space, reducing treatment complexity and extending the benefits of implantsupported restorations to a larger patient population. [6]. Mini-implants can be indicated for restoration of mandibular anterior teeth [11]. Various case studies suggested that the mini-implants can be used successfully for retaining mandibular overdenture [12]. Multiple teeth can be replaced by splinting mini-implant with a regular diameter implant. Also, mini-implants can be used for posterior single tooth replacement.
When used in high-stress bearing areas (posterior region), large amounts of cyclic loading may induce metal fatigue in the mini-implants. This fatigue may be due to the concentration of more forces per unit area of the implant body. Therefore, appropriate precautions are to be taken to control the occlusal forces by modifying the cusp or splinting the multiple mini-implants [13]. Additionally, they can be used in conjunction with larger diameter implants, and a splinted prosthesis is given for the uniform distribution of the loads [8]. Case studies with short-term follow-up were found to be satisfactory from the perspective of patient comfort and limited marginal bone loss $[6,14]$.

\section{Contraindications}

The mini-implants are contra-indicated in medically compromised patients with uncontrolled diabetes, coagulation disorders, chemotherapy, and radiotherapy. Also, they are not recommended for patients with systemic diseases associated with wound healing and bone healing. Mini-implants are also not indicated in patients with chronic periodontitis, limited soft tissue coverage, insufficient bone height, parafunctional

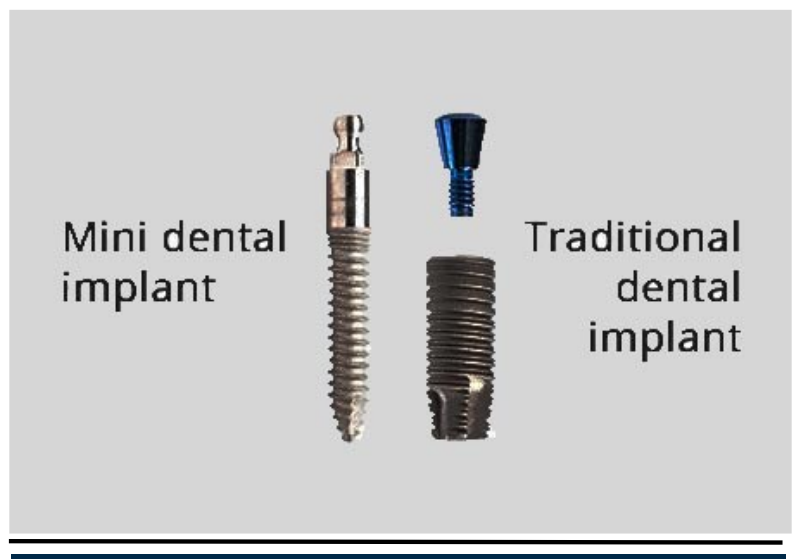

Figure 1. Design of a mini-implant

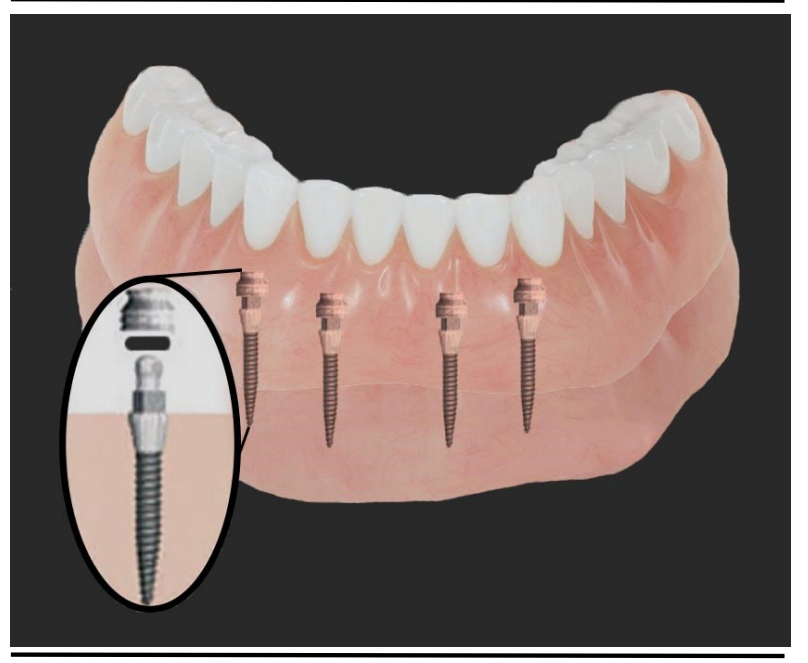

Figure 2. Mini-implant with 0-ring 
habits that cause predetermined vertical and lateral occlusal forces, and children until epiphyseal closure is complete $[6,15]$.

\section{Placement procedure}

Proper diagnostic data is essential and $O P G$ is a prerequisite for accurate presurgical planning and placement. Along with OPG, diagnostic models with radio-opaque markers $\mathrm{CBCT}$ are advised for $3 \mathrm{D}$ planning, particularly in very narrow ridges for proper planning to place the implant and angulation [15].

In the surgical procedure, crestal infiltration of local anaesthesia is adequate as the mini-implants are the least invasive [8]. Generally, raising a surgical flap is not required unless in a very narrow ridge with an abundant soft tissue where minimal flap raising is advocated after giving a crestal incision to visualize bone for precise implant placement with predetermined angulation [8].

During osteotomy, the use of a pilot drill is adequate [8]. Usually, osteotomy depth of one-third to one-half of the length of the chosen implant is sufficient for achieving primary stability [13].

\section{Insertion procedure}

The MDI (mini dental implant) is supplied in a glass vial suspended from a plastic cap (implant carrier) [14]. During insertion, the pouch is opened, and the implant is inserted into the osteotomy site. Then, the implant is torqued with a finger pressure in a clockwise direction with a downward direction until firm bony resistance is noticed to provide adequate primary stability [14]. To torque, the MDI ratchet wrench is used up to $40 \mathrm{NCM}$ in small carefully controlled torques [14]. During torquing, a finger is placed on top of the ratchet wrench to minimize non-axial forces and ensure the correct seating of the implant to its final depth [14]. At the end of insertion, the head of the MDI should protrude from the tissue without exposing threads [14].

\section{Insertion procedure}

Mini-implant-retained overdentures and crowns are generally subjected to immediate or progressive bone loading because of the one-piece design of the implant. According to Wolff's Law, gradual bone loading is associated with superior bone healing $[11,12]$. Various case studies demonstrated a negligible bone loss around the implants, radiographically [15].

\section{Advantages}

Mini-implants exhibit all the advantages similar to those of standard implants. As the name suggests that they are smaller in size, and they are minimally invasive. The inflammation is less in the implant site leading to faster healing and allows immediate loading. They require simple surgical procedures and causing minimum discomfort to the patient. There is less linear or circumferential percutaneous exposure of the implant resulting in less vulnerability to bacterial attack at the implant-gingival attachment. Thus, the characteristic resorption to the first thread phenomenon seen with regular diameter implants does not seem to be prevalent with these implants. Also, they require very minimal osteotomy preparation, which does not remarkably compromise angiogenesis $[1,5,9]$. In cases of the narrow residual ridge, mini-implants have become a choice of treatment. Innovation of miniimplants minimized the necessity of grafting procedures, ridge augmentation. They are economically viable when compared to standard implants. In case of failure, they are easy to remove with minimal surgical trauma [8].

\section{Limitations}

Mini-implants cannot be placed in ridges with inadequate vertical height and in an individual with parafunctional habits and cases with reduced inter arch space [15]. The potential for fracture of the implant during placement is more due to its narrow diameter [5]. There is a lack of parallelism between implants because of their one-piece design. There is a need for multiple implant placement because of failure unpredictability due to lack of scientific guidelines and understanding [15]. There is limited scientific evidence about the long-term survival of these implants [7]. The clinical success of mini-implant-supported fixed restorations requires a thorough understanding of biomechanics through proper case selection and precise treatment execution $[7,15]$.

\section{Conclusion}

Mini-implants are considered a feasible treatment option in appropriate circumstances based on the minimal scientific evidence and case reports available. Case selection becomes a significant criterion due to 
the small surface area of mini-implants leading to less bone-implant contact, which impacts the success of the implants. Stress variables also should be considered as prime aspects while placing mini-implants on a narrow ridge.

Conflicts of interest: Authors declared no conflicts of interest.

\section{Financial support: None}

\section{References}

1. Romeo E, Ghisolfi M, Rozza R, Chiapasco M, Lops D. Short (8-mm) dental implants in the rehabilitation of partial and complete edentulism: a 3- to 14-year longitudinal study. Int J Prosthodont. 2006;19(6):586592

2. Cardaropoli G, Lekholm U, Wennstrom JL. Tissue alterations at implant-supported single-tooth replacements: a 1-year prospective clinical study. Clin Oral Implants Res. 2006;17(2):165-171. https://doi.org/10.1111/j.1600-0501.2005.01210.x

3. Tarnow DP, Cho SC, Wallace SS. The effect of interimplant distance on the height of inter-implant bone crest. J Periodontol. 2000; 71(4):546-549. https://doi.org/10.1902/jop.2000.71.4.546

4. Esposito M, Grusovin MG, Coulthard P, Worthington HV. The efficacy of various bone augmentation procedures for dental implants: a Cochrane systematic review of randomized controlled clinical trials. Int $\mathbf{J}$ Oral Maxillofac Implant. 2006;21(5).696-710. https:// doi.org/10.1002/14651858.cd003607.pub2

5. Flanagan D. Fixed partial dentures and crowns supported by very small diameter dental implants in compromised sites. Implant Dent. 2008; 17(2):182-191. https://doi.org/10.1097/ID.0b013e31817776cf

6. Davarpanah M, Martinez H, TECUCIANU JF, Celletti R, Lazzara R. Small-diameter implants: Indications and contraindications. J Esthet Rest Dent. 2000 Jul;12(4):186-94. https://doi.org/10.1111/j.1708-8240.2000.tb00221.x

7. Froum SJ, Cho SC, Cho YS, Elian N, Tarnow D. Narrow-diameter implants: a restorative option for limited interdental space. Int $\mathrm{J}$ Periodont Rest Dent. 2007;27(5):449-455

8. Sohrabi K, Mushantat A, Esfandiari S, Feine J. How successful are small-diameter implants? A literature review. Clin Oral Implants Res 2012;23(5):515-525. https://doi.org/10.1111/j.1600-0501.2011.02410.x

9. Comfort MB, Chu FC, Chai J, Wat PY, Chow TW. A 5-year prospective study on small diameter screwshaped oral implants. J Oral Rehabil. 2005; 32(5):341 -345 .

https://doi.org/10.1111/j.1365-2842.2004.01441.x
10. Tarnow D, Elian N, Fletcher P, Froum S, Magner A, Cho SC, Salama M, Salama H, Garber DA. Vertical distance from the crest of bone to the height of the interproximal papilla between adjacent implants. J Periodontol. 2003; 74(12):1785-1788. https:// doi.org/10.1902/jop.2003.74.12.1785

11. Roberts WE, Garetto LP, DeCastro RA. Remodeling of devitalized bone threatens periosteal margin integrity of endosseous titanium implants with threaded or smooth surfaces: indications for provisional loading and axially directed occlusion. J Indiana Dent Assoc 1989;68(4):19-24.

12. Misch CE. Density of bone: effect on treatment plans, surgical approach, healing, and progressive bone loading. Int J Oral Implantol. 1990;6(2):23-3.

13. Kumari P, Verma M, Sainia V, Gupta A, Gupta R, Gill S. Mini-Implants, Mega Solutions: A Case Series. J Prosthodont. 2016;25(8):682-6. https:// doi.org/10.1111/jopr.12382

14. Vigolo P, Givani A, Majzoub Z, Oordioli G. Clinical evaluation of small-diameter implants in single-tooth and multiple-implant restorations: a 7-year retrospective study. Int J Oral Maxillofac Implant. 2004;19(5): 703-709.

15. Bidra AS, Almas K. Mini implants for definitive prosthodontic treatment: a systematic review. J Prosthet Dent. 2013;109(3):156-64. $\quad \underline{\text { https:// }}$ doi.org/10.1016/S0022-3913(13)60035-9 